\title{
Involving patients with dementia in decisions to initiate treatment: effect on patient acceptance, satisfaction and medication prescription
}

\author{
Jemima Dooley, Nick Bass, Gill Livingston and Rose McCabe
}

\section{Background}

Shared decision-making is advocated but may be affected by cognitive impairment. Measures of shared decision-making provide global descriptions of communication without detailed analysis of the subtle ways in which doctors invite patient input.

\section{Aims}

We aimed to explore medication decisions in dementia, using a standardised Treatment Recommendation Coding Scheme.

\section{Method}

We analysed 71 video-recorded dementia diagnostic meetings from nine memory clinics. Recommendations were coded as pronouncements ('I will start you on medication'), proposals ('Shall we try medication?'), suggestions ('Would you like to try medication?'), offers ('I can prescribe medication') or assertions ('There is medication'). Patient responses were coded as acceptance ('I'd like to have that'), active resistance ('I'm not very keen') and passive resistance (minimal or no response). cognitive test scores, prescription rates and satisfaction were assessed and associations were explored.

\section{Results}

Doctors used suggestions in $42 \%$ of meetings, proposals in $25 \%$, assertions in $13 \%$, pronouncements in $11 \%$ and offers in $9 \%$.
Over $80 \%$ of patients did not indicate clear acceptance. Patients were most likely to actively resist after suggestions. There was no association between cognitive impairment and recommendation format. Patients were less satisfied with pronouncements. Patient preference did not influence whether medication was prescribed.

\section{Conclusions}

Doctors initially nominate people with dementia as the decision maker, and this is unaffected by cognitive impairment. Over $80 \%$ of patients resisted starting medication, mostly through passive resistance, the most common form of disagreement in communication. Medication still tended to be prescribed, indicating that factors other than patient preference affect prescription.

\section{Declarations of interest}

None.

\section{Keywords}

Dementia; Alzheimer's disease; shared decision-making; medication decision; communication.

\section{Copyright and usage}

(c) The Royal College of Psychiatrists 2018

\section{Shared decisions in dementia}

Involving patients in decisions about their psychiatric treatment has been shown to increase patient satisfaction and treatment adherence and improve clinical outcomes. ${ }^{1}$ There has been little research observing how doctors communicate with people with dementia when making decisions about anti-dementia medication, ${ }^{2}$ but there is an indication that shared decision-making may be low. ${ }^{3}$ Cognitive impairment in dementia, alongside the increasing role of family, has been shown to affect patient involvement. ${ }^{4}$ However, as in other illnesses, people with dementia want to be involved in discussions about their care. ${ }^{5}$

\section{How (not whether) decisions are shared}

Most research on shared decision-making used scales that offer a global picture of patient involvement, such as whether doctors ask patient preferences or list options. ${ }^{6,7}$ However, analysis of how rather than whether doctors discuss medication with patients is vital for a more nuanced understanding. How doctors recommend treatment is particularly vital: it is at this point that the patient is identified either as the primary decision maker or a passive party. ${ }^{8}$ Recently, a novel approach examining how doctors format treatment recommendations in more or less authoritative ways has been developed. ${ }^{9}$ As Stivers and colleagues point out, there are clear differences between 'I'm going to start you on X', 'We can give you X to try' and 'Would you like me to give you X?'. As these different formats are more or less authoritative, they afford patients different degrees of autonomy to decide whether to accept or resist treatment recommendations. Furthermore, just as medication recommendations are subtly different, subtle differences in patient responses also reflect different degrees of acceptance or resistance. A well-established body of studies on agreement and disagreement in interaction have shown that although acceptance occurs quickly and directly, resistance occurs with delay and can be either passive or active. ${ }^{10-12}$ Passive resistance involves a delayed or withheld response, a minimal verbal response (e.g. ' $\mathrm{mhm}$ ') or a non-verbal response (e.g. a nod). ${ }^{10}$ Exposing disagreement is socially problematic, even more so in medical consultations given doctor authority, and thus most disagreement in communication takes the form of passive resistance. ${ }^{13}$ This social delicacy in disagreement means active resistance (explicit statements of non-agreement or questioning) occurs more infrequently. ${ }^{10}$

The aim of this study was to examine how doctors involve patients with dementia in decisions to start medication through a detailed analysis of the subtle ways in which doctors invite patient input and how this affects patient acceptance of medication. Associations with patient cognitive function, satisfaction and prescription were also explored.

\section{Method}

The Camden and Islington National Research Ethics Committee approved the project (13/LO/1309). The data was collected as part of the Shared decision making in mild to moderate Dementia study (ShareD), ${ }^{3}$ funded by the National Institute for Health 
Research, Research for Patient Benefit (PB-PG-1111-26063). This was an observational study collecting video recordings from routine memory clinic diagnostic feedback appointments where patients were told whether they had dementia. Recruitment was conducted in two sites in the UK: London (urban) and Devon (rural). In London, there were six participating memory clinics across three National Health Service trusts. In Devon, there were three participating memory clinics within one National Health Service trust. Recruitment ran from May 2014 to July 2016.

\section{Recruitment}

We approached all clinicians delivering dementia diagnoses in the participating trusts. The aim was to recruit all consecutive patients attending the memory clinic for diagnosis feedback, except for those needing interpreters because of the added complexity of the communication. Administrative staff sent information sheets with patient appointment letters. Clinicians assessed whether patients had capacity to provide informed consent, and researchers approached patients and their companions to discuss the study. Two patients in this study were judged not to have capacity, and so we followed the Department of Health guidance on nominating a consultee for research involving adults who lack the capacity to consent. ${ }^{14}$

\section{Data collection}

\section{Treatment recommendations and responses}

We filmed patient meetings with clinicians by GoPro camera, without the researcher present. The meetings were transcribed verbatim and medication discussions identified. Detailed conversation analysis transcription methods were used for the excerpts of the meeting containing treatment recommendations and responses to illustrate characteristics of speech, such as pauses, overlap, stress, intonation and pace. ${ }^{15}$ For clarity, the markers for prosody, stress and speed have been removed, leaving the markers for the overlapping speech (represented by square brackets) and length of silences (represented in seconds in brackets, with full stops representing pauses under $0.2 \mathrm{~s}$ ). The detailed transcripts are available from the authors on request.

\section{Patient capacity}

Patient capacity to make decisions about medication was recorded by clinicians after the meeting. Capacity was recorded as full, partial or no capacity.

\section{Cognitive test score}

Cognitive functioning was assessed by clinicians, using the Addenbrooke's Cognitive Examination - $\mathrm{III}^{16}$ or Mini-Mental State Examination cognitive test, ${ }^{17}$ as part of usual practice.

\section{Patient satisfaction}

After the meeting the patient completed the Patient Experience Questionnaire. $^{18}$ The communication subscale of the Patient Experience Questionnaire was used to obtain communication-specific satisfaction ratings. This subscale contains four statements ('We had a good talk', 'I felt reassured', 'The doctor understood what was on my mind' and 'I felt I was taken care of), each with a five-point Likert scale from 'agree completely' to 'disagree completely'. The total possible score is 20 .

\section{Prescription outcome}

Whether medication was prescribed was identified from the recordings. The reason for not prescribing medication was recorded.

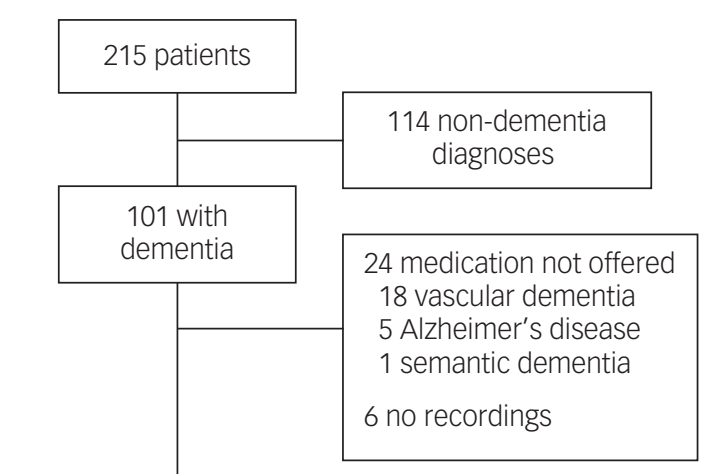

71 medication recommendations

Fig. 1 Patient flow diagram.

\section{Data analysis}

\section{Treatment recommendation}

Each recommendation was coded by the Treatment Recommendation Coding Scheme, ${ }^{9}$ which was developed in primary care (UK and USA), psychiatry (UK) and neurology (UK) settings. The coding scheme was comprehensively developed with conversation analysis methods that inductively examine how treatment recommendations are made in practice.

The coding scheme includes five recommendation formats, as follows: pronouncements, where patients are given no choice (e.g. 'I will start you on medication'); proposals, where patients are invited to endorse or collaborate with the doctor's idea (e.g. 'How about trying medication?'); suggestions, where medication is endorsed by doctors but patients are given the choice (e.g. 'Would you like to try medication?'); offers, where doctors show willingness to prescribe for the patient but do not actively endorse medication (e.g. 'Do you want me to give you medication?') and assertions, where doctors state the fact that medication exists without endorsement or explicit recommendation (e.g. 'There is a medication').

\section{Patient responses}

Using the coding scheme, the patient response was coded as follows: acceptance, namely quick positive acceptance (e.g. 'I'd like to have that'); passive resistance, namely minimal verbal or non-verbal acknowledgment (e.g. 'mhm' or nodding, or no response) or active resistance, namely questioning the purpose of medication or indicating a desire not to take the medication (e.g. 'I'm not very keen, I don't want to take more tablets').

Two researchers coded all the recommendation formats and patient responses and discrepancies were resolved through discussion. Relationships between treatment recommendation, patient acceptance or resistance, and whether medication was prescribed were explored by Fischer's exact tests. Possible associations between recommendation format and patient acceptance or resistance, cognitive test score and patient satisfaction were explored by analysis of variance. Researchers coding treatment recommendations were blind to patient capacity and whether medication was prescribed.

\section{Results}

The consent rate for clinicians in the study was $88 \%$. Of the 215 patient participants (consent rate 51\%), 101 were diagnosed with dementia. Of the 101 video-recorded meetings where patients 


\begin{tabular}{|c|c|}
\hline Characteristic & $N(\%)$ \\
\hline \multicolumn{2}{|l|}{ Site } \\
\hline London & $30(42)$ \\
\hline Devon & $41(58)$ \\
\hline \multicolumn{2}{|l|}{ Patient gender } \\
\hline Female & $44(62)$ \\
\hline Patient age, mean (range) & $81(65-91)$ \\
\hline \multicolumn{2}{|l|}{ Patient ethnicity } \\
\hline White British/Irish & $59(82)$ \\
\hline White other & $3(4)$ \\
\hline Caribbean & $3(4)$ \\
\hline Black or Black British & 1 (2) \\
\hline African & 1 (2) \\
\hline Other & 2 (3) \\
\hline Missing & 2 (3) \\
\hline \multicolumn{2}{|l|}{ Patient diagnosis } \\
\hline Alzheimer's disease & $49(69)$ \\
\hline Mixed dementia & $14(20)$ \\
\hline Dementia unspecified & $4(5)$ \\
\hline Parkinson's disease & 2 (3) \\
\hline Lewy body dementia & 2 (3) \\
\hline \multicolumn{2}{|l|}{ Patient Cognitive Test score, mean (range) } \\
\hline Addenbrooke's Cognitive Examination - III ( $n=58)$ & $69(41-94)$ \\
\hline Mini-Mental State Examination $(n=11)$ & $23(15-28)$ \\
\hline \multicolumn{2}{|l|}{$\begin{array}{l}\text { Patient capacity to make decision about medication } \\
\text { (judged by doctor) }\end{array}$} \\
\hline Full & $43(61)$ \\
\hline Partial & $16(22)$ \\
\hline None & $3(4)$ \\
\hline Missing & $9(13)$ \\
\hline Companion present & $67(94)$ \\
\hline \multicolumn{2}{|l|}{ Companion type } \\
\hline Spouse/partner & $27(40)$ \\
\hline Child/child in law & $27(40)$ \\
\hline Sibling & $2(3)$ \\
\hline Friend & $2(3)$ \\
\hline Other & $8(12)$ \\
\hline Missing & 1 (2) \\
\hline \multicolumn{2}{|l|}{ Professional type } \\
\hline Consultant psychiatrist & $15(71)$ \\
\hline Consultant geriatrician & $3(14)$ \\
\hline Specialty doctor & $3(15)$ \\
\hline $\begin{array}{l}\text { Professional number of years working in dementia, } \\
\text { mean (range) }\end{array}$ & $12(1-25)$ \\
\hline \multicolumn{2}{|l|}{ Professional gender } \\
\hline Female & $11(52)$ \\
\hline \multicolumn{2}{|l|}{ Ethnicity } \\
\hline White British & $14(66)$ \\
\hline White other & $3(14)$ \\
\hline Asian or Asian British & $2(10)$ \\
\hline Indian & $2(10)$ \\
\hline
\end{tabular}

received a diagnosis of dementia, 71 were included (Fig. 1). In the remaining 30 recordings there were six cases of equipment malfunction, 18 vascular dementia diagnoses where medication was not offered and one semantic dementia diagnosis where medication was not offered. In five patients with Alzheimer's disease, medication was not recommended: either medication was not discussed at all, or it was discussed but not presented as an option.

\section{Participant characteristics}

Participant characteristics are displayed in Table 1. There were 71 patients, 67 companions and 21 doctors. Most patients were White and received a diagnosis of Alzheimer's disease or mixed dementia. Nearly two-thirds were female. Where doctor judgement of patient capacity to make medication decisions was recorded, 70\% had full and $22 \%$ had partial capacity. Companions were present in most meetings and were nearly always spouses/partners or children/ children in law. Doctors were primarily psychiatrists, with three geriatricians.

\section{Treatment recommendation formats and responses Treatment recommendation formats}

Doctors primarily formatted treatment recommendations in a way that nominated the patient as the primary decision maker. In $73 \%$ of treatment discussions, doctors initiated the discussion with assertions, i.e. a general statement about the existence of medication such as 'There are medications we can try'. These preliminary statements usually either contained a reference to the tablets not being a cure and/or a description of side-effects. In the majority of meetings where discussions started with assertions, these assertions were treated as information giving: there was little response from patients and doctors quickly moved on to the treatment recommendation proper. The first statement by the doctor in Extract 1 is an example of this.

\section{Extract 1:}

Doctor: Now (0.6) there is some medication (.) that (0.3) many people take (.) to help with the symptoms of memory loss $(0.5)$

Doctor: And about two-thirds of people find it gives them some benefit (0.4)

Doctor: Uhm some people most people don't but some people get some side-effects such as a little bit of nausea or a loose stool or a headache or a feeling of dizziness (0.6)

Doctor: Would you like to think about taking some medication? (0.6)

Patient: Yes well (0.9), see how it goes. I've got a lot of medication I take almost every day.

The doctor begins by asserting that there is 'some medication' to help memory loss, followed by the caveats that not all people will benefit and that some people get some side-effects. This is followed by a suggestion, where the doctor nominates the patient as the decision maker: 'Would you like to think about taking some medication?'.

Suggestions, as in Extract 1, were used in $42 \%$ of meetings. Proposals were used in $25 \%$ of meetings ('We could try you on a tablet to help contain or maintain or make this stable in the future'), pronouncements in $11 \%$ of meetings ('I would want you to start at least taking medication') and offers in $9 \%$ of meetings ('The other thing we can do is to give you a tablet if you would like?'). Assertions were used as the only treatment recommendation type in $13 \%$ ('There is now medications that we can offer people').

\section{Patient responses}

Forty-five patients (63\%) passively resisted medication by responding minimally (e.g. ' $m$ hm'), nodding or not responding; 13 patients (18.5\%) actively resisted medication ('Is it going to help, I take medication already?') and 13 patients (18.5\%) explicitly accepted medication ('Yes I'll take them').

\section{Association between recommendation format and patient response}

There was a significant association between recommendation format and patient response (Fisher's exact test $P=0.014$ ). Patients only actively resisted medication after suggestions or proposals, and always passively resisted pronouncements (see Table 2). In just over half of the cases where patients accepted medication, doctors had used proposals. 
Table 2 Treatment recommendation, response and prescription outcomes

\begin{tabular}{lcrr|} 
& Acceptance & Passive resistance & Active resistance \\
Pronouncement & 0 & 8 & 0 \\
Proposal & 7 & 9 & 2 \\
Suggestion & 3 & 16 & 11 \\
Offer & 1 & 5 & 0 \\
Assertion & 2 & 7 & 0 \\
Total & 13 & 45 & 9 \\
\hline Prescribed & 9 & 36 & 9 \\
Not prescribed & 4 & 9 & 9 \\
\hline
\end{tabular}

\section{Recommendation format and cognitive test score}

There was no association between recommendation format and the patient's level of cognitive impairment, as shown on the Addenbrooke's Cognitive Examination - III $(F(4,53)=0.478, P=0.751)$ or MiniMental State Examination $(F(3,7)=0.557, P=0.660)$.

\section{Recommendation format and patient satisfaction}

Patients were significantly less satisfied with the communication when they were not offered a choice in taking medication, i.e. when pronouncements were used (14.3/20) compared with other recommendation formats $(16.5-17.3 / 20)(F(4,59)=3.047, P=0.024)$.

\section{Patient response and prescription outcomes}

Medication was prescribed in $76 \%(n=54)$ of the meetings. There was no association between patient acceptance or resistance and whether medication was prescribed (Fisher's exact test $P=0.561$ ). Medication was just as often prescribed when patients resisted as when they accepted (see Table 2).

For the 17 patients where medication was not prescribed, nine (53\%) decisions were deferred for further investigation, either an electrocardiogram or referral to neurology. Four (24\%) patients expressed a wish to talk to family or their general practitioner before making a decision, and for two patients (11.5\%) there was a joint decision not to take medication. For the remaining two patients $(11.5 \%)$ the doctor decided to defer the decision until support was in place from the community mental health team.

\section{Discussion}

Doctors in memory clinics overwhelmingly nominated patients as the decision maker when starting medication for dementia. Over $80 \%$ of patients appeared to show some reluctance to start medication, primarily through passive resistance reflecting the most common form of disagreement in communication. However, medication still tended to be prescribed.

The use of assertions to initiate medication discussions has also been described in neurology. ${ }^{19}$ Assertions are poised between informing the patient about medication and recommending medication to the patient. This places very little interactional pressure on the patient for an immediate decision, thus allowing for further discussion. Toerien describes assertions as 'cautious recommendations' in environments of diagnostic uncertainty, including uncertain efficacy of the mediation and potential patient resistance. ${ }^{19}$ In memory clinics, patients will be dealing with a life-changing diagnosis of dementia. The use of assertions before an explicit recommendation may therefore facilitate a smoother, more sensitive transition between the diagnosis and treatment decisionmaking. Assertions also inform the patient about the likely benefits and side-effects of medication, as it can be difficult to agree to take a new medication before having this information.
Doctors used fewer pronouncements than in primary care $(65 \%$ USA, $45 \% \mathrm{UK})^{9}$ and general psychiatry $(25 \%){ }^{20}$ This suggests a higher willingness to include patients in medication decisions at dementia diagnosis. Indeed, two-thirds of treatment recommendations were suggestions or proposals, demonstrating that doctors endorse medication as a treatment option but are encouraging patient participation in the decision. However, subtle differences in the precise formulation of the recommendation have an effect on patient response. With proposals, doctors are inviting patients to join in with their endorsement of medication. With suggestions, doctors are inviting the patient to decide whether they would like to start medication. ${ }^{9}$ This has important consequences for how patients respond: proposals lead to higher levels of acceptance whereas suggestions lead to higher levels of resistance. This demonstrates how subtly different formats create different possibilities for patient choice and participation in decision-making.

The majority of patients appeared to show some reluctance to start medication. However, previous work on resistance to treatment recommendations has been done with patients who are cognitively intact in primary care and patients with psychosis in out-patient psychiatric care. It may be that passive resistance - a non-verbal response or a minimal verbal response - in people who are cognitively intact does not indicate passive resistance in people who are cognitively impaired. For example, there is evidence that reaction times in conversation can be five times slower for people with moderate dementia than people without dementia. ${ }^{21}$ Therefore, people with dementia may be responding more slowly when withholding a response rather than resisting. Conversely, people with dementia have also been found to agree more in conversation, suggesting an attempt to remain engaged in the interaction through assent, rather than actual agreement. ${ }^{22}$

Although marginalisation of people with dementia has been described in previous studies, ${ }^{2,23,24}$ doctors involved patients in decisions irrespective of cognitive impairment. This is a positive finding, demonstrating that doctors in UK memory clinics are not excluding patients with lower cognitive test scores from decisions about their medication, and is in line with patients' preferences to be involved in decision-making. ${ }^{4,5}$ However, the majority of patients in this study were in mild to moderate stages of dementia, and decision-making with people in later stages may be different.

Patients were significantly less satisfied with communication when they are not offered a choice ('I'll start you on medication'), illustrating that patients detect subtle differences in communication. This is in line with other studies of shared decision-making ${ }^{25}$ and demonstrates the importance of including people with dementia in decisions. However, it must be noted that patients were rating their satisfaction with communication in the meeting as a whole, and there could be other communication difficulties affecting patient experience and potentially causing doctors to use pronouncements when it came to recommending medication.

Patients' acceptance or resistance of medication did not influence whether medication was prescribed. In $69 \%$ of cases where patients 
actively resisted medication (nine out of 13), it was nonetheless prescribed. If medication was not prescribed, this was usually because of a need for further investigation (electrocardiogram or neurology) or doctors planning to put other support systems in place before starting medication. However, there are other factors that are likely to come into play. In this study, $8 \%$ of patients were judged not to have capacity to make medication decisions and $22 \%$ were judged to have partial capacity. Moreover, doctors report wishing to offer medication to instil hope after breaking bad diagnostic news, and therefore may find it difficult not to prescribe medication. ${ }^{26}$ Family preferences may also come into play: doctors have been shown to invite family involvement in medication discussions more than in other parts of the consultation, ${ }^{27}$ and caregivers deem medication to be important for access to services and to provide hope for the future. ${ }^{28}$ However, it is worth noting that only the patient's initial response to the treatment recommendation was taken into account in this analysis, and it may be that they would have changed their minds after further discussion.

\section{Strengths and limitations}

The strength of this study was the systematic standardised analysis of treatment recommendations, using a novel approach to capture more subtle aspects of shared decision-making. This is relevant both to oldage psychiatry and other areas of psychiatry where patients may experience cognitive impairment, such as schizophrenia or intellectual disabilities. This study is the first to use this detailed method to examine decision-making when people receive a diagnosis of dementia, and includes a range of patients, doctors and clinics. When coding the treatment recommendations, researchers were blind to patient capacity and whether medication was prescribed. However, doctors knew they were being filmed and this may have affected their behaviour. Companion behaviour was not analysed, which may have affected decision-making. Additionally, the majority of the patient were White, and consultations with an interpreter were not included, which limits generalisability.

\footnotetext{
Jemima Dooley, PhD (iD), Research Associate in Qualitative Methods, Population Health Sciences, Bristol Medical School, University of Bristol, UK; Nick Bass, MD, Senior Clinical Lecturer and Honorary Consultant, Division of Psychiatry, University College London, UK: Lecturer and Honorary Consultant, Division of Psychiatry, University College Lond
Givingston, FRCPsych, Professor of Psychiatry of Older People, Division of Psychiatry, University College London, UK; Rose Mccabe, PhD, Professor of Clinical Communication, Health Services Research, University of Exeter Medical School, UK

Correspondence: Jemima Dooley, Population Health Sciences, Bristol Medical School, Room 1.10, Canynge Hall, 39 Whatley Road, Bristol BS82PS, UK.

Email: jemima.dooley@bristol.ac.uk

First received 25 Jan 2018, final revision 12 Jun 2018, accepted 28 Aug 2018
}

\section{Funding}

This paper presents independent research funded by the National Institute for Health Research (NIHR) under its Research for Patient Benefit programme (grant PB-PG-1111-26063), and is supported by the NIHR Collaboration for Leadership in Applied Health Research and Care South West Peninsula. The views expressed are those of the authors and not necessarily those of the National Health Service, the NIHR or the Department of Health.

\section{Acknowledgements}

We thank all the patients, companions and healthcare professionals who allowed us to film the consultations, as well as the ShareD project team.

\section{References}

1 McCabe R, Priebe S. The therapeutic relationship in the treatment of severe mental illness: a review of methods and findings. Int J Soc Psychiatry 2004; 50 (2): $115-28$.

2 Dooley J, Bailey C, McCabe R. Communication in healthcare interactions in dementia: a systematic review of observational studies. Int Psychogeriatr 2015; 27(8): 1277-300.
3 Mccabe R, Dooley J, Bailey C. Communicating a diagnosis of dementia. RCPsych Old Age Scientific Meeting, Bristol, 23 March 2017.

4 Miller L, Whitlatch C, Lyons K. Shared decision-making in dementia: a review of patient and family carer involvement. Dementia 2016; 15(5): 1141-57.

5 Hamann J, Bronner K, Margull J, Mendel R, Diehl-Schmid J, Bohner M, et al. Patient participation in medical and social decisions in Alzheimer's disease. J Am Geriatr Soc 2011; 59(11): 2045-52.

6 Pellerin M, Elwyn G, Rousseau M, Stacey D, Robitaille H, Legare F. Toward shared decision making: using the OPTION scale to analyse resident-patient consultations in family medicine. Acad Med 2011; 86(8): 1010-8.

7 McCabe R, Khanom H, Bailey P, Priebe S. Shared decision-making in ongoing outpatient psychiatric treatment. Patient Educ Couns 2013; 91(3): 326-8.

8 Elwyn G, Hutchings H, Edwards A, Rapport F, Wensing M, Cheung W, et al. The OPTION scale: measuring the extent that clinicians involve patients in decision making tasks. Health Expect 2005; 8: 34-42.

9 Stivers T, Heritage J, Barnes R, McCabe R, Thompson L, Toerien M. Treatment recommendation as actions. Health Commun 2017; 33(11): 1335-44.

10 Stivers T. Parent resistance to physicians' treatment recommendations: one resource for initiating negotiation of the treatment decision. Health Commun 2005; 181(1): 41-74.

11 Ijas-Kallio T, Ruusuvuori J, Perakyla A. Patient resistance towards diagnosis in primary care: Implications for concordance. Health (London) 2010; 14(5): 505-22.

12 Heritage J, Selfi S. Dilemmas of advice: aspects of the delivery and reception of advice in interactions between health visitors and first time mothers. In Talk at Work: Interaction in Institutional Settings (eds P Drew, J Heritage): 359-417. Cambridge University Press, 1992.

13 Pomerantz A. Agreeing and disagreeing with assessments: some features of preferred and dispreferred turn shapes. In Structures of Social Action (ed. JM Atkinson, J Heritage): 57-101. Cambridge University Press, 1984.

14 Department of Health. Guidance on Nominating a Consultee for Research Involving Adults Who Lack Capacity to Consent. TSO, 2008 (http://webarchive. nationalarchives.gov.uk/20130123193236/http://www.dh.gov.uk/en/ Publicationsandstatistics/Publications/PublicationsPolicyAndGuidance/ DH_083131).

15 Jefferson G. Glossary of transcript symbols with an introduction. In Conversation Analysis: Studies from the First Generation (ed. GH Lerner): 13-31. John Benjamins, 2004.

16 Hsieh S, Schubert S, Hoon C, Mioshi E, Hodges JR. Validation of the Addenbrooke's cognitive examination III in frontotemporal dementia and Alzheimer's disease. Dementia and Geriatric Cognitive Disorders 2013; 36 242-50.

17 Arevalo-Rodriguez I, Smailagic N, Roqué I Figuls M, Ciapponi A, SanchezPerez E, Giannakou A, et al. Mini-Mental State Examination (MMSE) for the detection of Alzheimer's disease and other dementias in people with mild cognitive impairment (MCI). Cochrane Database Syst Rev 2013; 3: CD010783.

18 Steine $S$, Finset A, Laerum E. A brief new questionnaire (PEQ) developed in primary healthcare for measuring patients' experience of interaction, emotion and consultation outcome. Family Practice 2001; 18: 410-8.

19 Toerien M. Deferring the decision point: treatment assertions in neurology outpatient consultation. Health Commun 2018; 33(11): 1355-65.

20 Thompson L, McCabe R. How psychiatrists recommend treatment and its relationship with patient uptake. Health Commun 2018; 33(11): 1345-54.

21 Jones G. A communication model for dementia. In Care-Giving in Dementia: Volume 1: Research and Applications (eds G Jones, BML Miesen): 78-99. Routledge, 1992.

22 Sugarman J, Roter D, Cain C, Wallace R, Schmechel D, Welsh-Bohmer KA Proxies and consent discussions for dementia research. J Am Geriatr Soc 2007; 55(4): 556-61.

23 Karnieli-Miller O, Werner P, Aharon-Peretz J, Sinoff G, Eidelman S. Expectations, experiences, and tensions in the memory clinic: the process of diagnosis disclosure of dementia within a triad. Int Psychogeriatr 2012; 24(11): 1756-70.

24 Hasselkus BR. Three track care: older patient, family member and physician in the medical visit. J Aging Stud 1994; 8(3): 291-307.

25 Hamann J, Leucht S, Kissling W. Shared decision making in psychiatry. Acta Psychiatr Scand 2003; 107: 413-09.

26 Bailey C, Dooley J, McCabe R. 'How do they want to know?' Doctors' perspectives on making and communicating a diagnosis of dementia. Dementia, 2018; DOI: 10.1177/1471301218763904

27 Karnieli-Miller O, Werner P, Neufeld-Kroszynski G, Eidelman S. Are you talking to me?! An exploration of the triadic physician-patient-companion communication within memory clinic encounters. Patient Educ Couns 2012; 88: 381-90.

28 Campbell S, Manthorpe J, Samsi K, Abley C, Robinson L, Watts S, et al. Living with uncertainty: mapping the transition from pre-diagnosis to a diagnosis of dementia. J Ageing Stud 2016; 37: 40-7. 